Original Research Paper

\title{
Microwave Assisted Regioselective Synthesis and Biological Evaluation of Pyrano[2,3-c]Pyridine Derivatives Utilizing DMAP as a Catalyst
}

\author{
Asmaa M. Fahim \\ Department of Green Chemistry, National Research Center, Dokki, P.O. Box.12622 Cairo, Egypt
}

Article history

Received: 02-10-2017

Revised: 06-12-2017

Accepted: 14-12-2017

E-mail: asmaamahmoud8521@hotmail.com asmaamahmoud8521@gmail.com

\begin{abstract}
Regioselective facile production of pyrano[2,3-c]pyridine through multicomponent reaction of aromatic aldehydes, ethyl cyano acetate or malononitrile and C- $H$ activated compound of 3-hydroxy picolinic acid in the occurrence of smaller amount of DMAP catalyst utilizing microwave apparatus, which is green and simple environmentally with high yield, recyclability catalyst. Totally the products were partitioned for antimicrobiogical action; it was detected that were active in contrast to S. pneumonia, E. coli and Candida albicans such as equated to typical drugs. Compounds of pyrano[2,3-c]pyridine-8-carboxylic acid derivatives $\mathbf{4 i}, \mathbf{4 e}, \mathbf{4 p}$ and $\mathbf{4 p}$ demonstrated effective development inhibitory activities. Additionally, the manufactured products were partitioned for in vitroantioxidant action by DPPH analysis. Products of pyrano[2,3-c]pyridine 40 and $4 \mathbf{p}$ were worthy free radical scavenging action through $\mathrm{IC}_{50}$ values of 252.52 and $223.2 \mu \mathrm{M}$; respectively.
\end{abstract}

Keywords: Multicomponent Reactions (MCR), 4-Dimethylaminopyridine (DMAP), Pyrano[2,3-c]Pyridine, Antimicrobial and Antioxidant Activity

\section{Introduction}

Multicomponent Reactions (MCR) have attempted extensive thought in combinatorial and biological chemistry (Thomas, 2017; Zhu, 2003; Zhu and Bienayme, 2005; Dömling, 2006; Dömling and Ugi, 2000). We established successfully numerous catalytic agent in organic synthesis exhausting MCR approach (Karnakar et al., 2015). The catalyst attractiveness is decrease of solvents ratios (Khan et al., 2008; 2010a; Khan 2010b and Khan, 2011) and furthermore performance role in the yield of the product. This would be inexpensive, mild and environmentally friendly for attention to the synthetic organic researcher. Dimethyl Amino Pyridine (DMAP) is a catalyst of outstanding effectiveness in a variation group-transfer reactions and considered for applications in stereo selective catalysis (Armand et al., 2014).

Pyrane and fused $4 H$-pyrane derivatives have concerned of interest (Dean, 1963) outstanding to their varied physiological activities. (Feuer, 1974) Earlier studies have presented that pyran derivatives possess pronounced chemical and biological activities, such antimicrobial activity, (Bonsignore et al., 1993; Ashraf, 2012) anti-coagulant, (Akbar et al., 2015; Dinesh et al.,
2017) anti-tumor and anti-HIV. Additionally, their besides valuable for the neurodegenerative disorder behavior, for instance Alzheimer, lateral amyotrophic sclerosis, Huntington's and Parkinson diseases (Fan et al., 2010) Moreover, they are similarly used as cosmetics, (Vyas et al., 2009) pigments, doze and useful as photoactive fabric (Nandakumar et al., 2010). In recent times, a limited approaches have been informed by hiring three-constituent responses exhausting different catalyst like as DBU (1,8-Diazabicyclo[5.4.0]undec-7ene) (Wen et al., 2001), TBAB(Tetra- $n$-butylammonium bromide) (Yusuke et al., 2016), ammonium phosphate (Saeed et al., 2015; 2007; Jitender and Ankita, 2012) hetero-poly supermans (Brahmachari et al., 2014). However, these procedures informed through, others are relatively beneficial, stable, there is auxiliary opportunity to improve innovative approach exhausting inexpensive catalyst above mild reaction stipulation and appropriate to a widespread variety of substrates.

\section{Materials and Methods}

\section{General Instruments}

Gallenkamp melting point apparatus were used for measuring the melting points. Furthermore the instrument 
Shimadzu FT-IR 8101 PC infrared spectrophotometer was used to record the IR spectrum. The ${ }^{1} \mathrm{H}-\mathrm{NMR}$ and ${ }^{13} \mathrm{C}$ NMR signals were evaluated in Deuterated Chloroform $\left(\mathrm{CDCl}_{3}\right)$ or DEUTERATED DIMETHYL SULFOXIDE $\left(\mathrm{DMSO}-d_{6}\right.$ ) at $300 \mathrm{MHz}$ on a Varian Mercury-VX 300 NMR spectrometer $\left({ }^{1} \mathrm{H}\right.$ at $300 \mathrm{MHz},{ }^{13} \mathrm{C}$ at $\left.75 \mathrm{MHz}\right)$ exhausting Trimethylsilane (TMS) as an interior signal. Shimadzu GCMS-QP 1000 EX mass spectrometer was used for detect the mass spectra at $70 \mathrm{eV}$. Elemental analyses were supported through Micro-analytical Center of Cairo University, Giza, Egypt. CEM Discover TM microwave instrument used for Microwave experiments.

\section{Material and Reagents}

3-hydroxypicolinicacid, methylbenzaldehyde, 4-chlorobs methoxybenzaldehyde, formaldehyde, isonicotine aldehyde from Aldrich Chemical CO. Ethanol and piperidine acquired from AldrichCompany. Methanol, petroleum ether; chloroform where BDH chemical reagents.

\section{Synthesis}

\section{Thermal Method}

Different of aromatic aldehydes (1mmol) and malononitrile, ethyl cyanoacetate $(1 \mathrm{mmol})$ in $4 \mathrm{ml}$ of ethanol was supplementary the catalyst DMAP $(0.025 \mathrm{~g}$, $0.2 \mathrm{mmol}$ ) and reserved magnificent at room tempeture. The obtained participation was formed instantaneously take 30-45 min in case of ethyl cyanoacetate while in malononitrile take few minutes and checked via Thin Layer Chromatography (TLC) and formerly allowable to relax at ordinary temperature, then Recrystallization from suitable solvent.

\section{Microwave Method}

Solution of aromatic aldehydes (1mmol) and malononitrile, ethyl cyano-acetate $(1 \mathrm{mmol})$ in $4 \mathrm{ml}$ of ethanol was additional the catalyst DMAP $(0.025 \mathrm{~g}$, $0.2 \mathrm{mmol}$ ) were diversified in Plus process vessel HP-500. The vessel was persevered accurately and irradiated through microwave underneath under pressure environments (17.2 bar, $100^{\circ} \mathrm{C}$ ) (Elham et al., 2014; Salem et al., 2015) assumed for 1-5 min with or without stirring After 5 min one-time the reaction mix was transformed to pure solution, the preceipted product approached out underneath hot condition at the required period mention in Table 2 (tested by TLC), The reaction mix was transported to typical temperature and formed precipitated was strained off to acquire the preferred products $\mathbf{4 a - 4 p}$.

\section{2-Amino-3-(Ethoxycarbonyl)-4-Phenyl-4H-} Pyrano[2,3-c]Pyridine-8-Carboxylic Acid (4a)

$\mathrm{C}_{18} \mathrm{H}_{16} \mathrm{~N}_{2} \mathrm{O}_{5}$ (340.11), Reddish brown $\left(230-232^{\circ} \mathrm{C}\right)$, Elemental analysis: C: 63.53(63.55), H: 4.74(4.75), N: $8.23(8.22)$, IR (KBr) $\bar{V}_{\max } / \mathrm{cm}^{-1}: 3410(\mathrm{OH}), 3310-$
3290 $\left(\mathrm{NH}_{2}\right)$ 1755(C= O). ${ }^{1} \mathrm{HNMR}\left(\mathrm{DMSO}-d_{6}\right): \delta 1.42(\mathrm{t}$, $\left.3 \mathrm{H}, H_{3} \mathrm{C}, J=3.1 \mathrm{~Hz}\right), 4.23\left(\mathrm{q}, 2 \mathrm{H}, H_{2} \mathrm{C}, J=3.1 \mathrm{~Hz}\right), 4.65$ $(\mathrm{s}, 1 \mathrm{H}, \mathrm{HC}), 6.72\left(\mathrm{~s}, 2 \mathrm{H}, \mathrm{H}_{2} \mathrm{~N} \mathrm{D} \mathrm{D}_{2} \mathrm{O}\right.$ exchangeable), 7.21$7.33(\mathrm{~m}, 5 \mathrm{H}, H \mathrm{C}$ aromatic), $8.10(\mathrm{~d}, 1 \mathrm{H}, H \mathrm{C}, J=12 \mathrm{~Hz})$, $8.58(\mathrm{~d}, 1 \mathrm{H}, H \mathrm{C}$ aromatic, $J=12 \mathrm{~Hz}), 12.025(\mathrm{~s}, 1 \mathrm{H}, H \mathrm{O}$ acid, $\mathrm{D}_{2} \mathrm{O}$-exchangeable), ${ }^{13} \mathrm{C}$ NMR (DMSO- $\left.d_{6}\right): \delta 14.2$ $\left(\mathrm{CH}_{3}\right), 43.2(\mathrm{CH}), 62.3\left(\mathrm{CH}_{2}\right), 79.2(\mathrm{CH}), 126.1(\mathrm{CH})$, $129.6(\mathrm{CH}), \quad 133.5(\mathrm{CH}), \quad 136.55(\mathrm{CH}), \quad 138.6(\mathrm{CH})$, 158.8 $(\mathrm{CH}), 168(\mathrm{C}=\mathrm{O}), 170(\mathrm{C}=\mathrm{O}) \mathrm{MS}(\mathrm{m} / \mathrm{z}$, aband.\%): $340\left(\mathrm{M}^{+}, 100 \%\right), 263(35.5 \%), 255(44.2 \%), 77(11.2 \%)$.

\section{2-Amino-3-Cyano-4-Phenyl-4H-Pyrano[2,3- c]Pyridine-8-Carboxylic Acid (4b)}

$\mathrm{C}_{16} \mathrm{H}_{11} \mathrm{~N}_{3} \mathrm{O}_{3}$ (293.28), Dark brown $\left(225-226^{\circ} \mathrm{C}\right)$, Elemental analysis: $\mathrm{C}$ : 65.53(65.55), H: 3.78(3.77), N: 14.33(14.35), IR (KBr) $\bar{V}{ }_{\text {max }} / \mathrm{cm}^{-1}: 3520(\mathrm{OH}), 3300-$ $3330\left(\mathrm{NH}_{2}\right), \quad 2230(\mathrm{C} \equiv \mathrm{N}) .{ }^{1} \mathrm{HNMR} \quad\left(\mathrm{DMSO}-d_{6}\right): \delta$ 4.51(s, $1 \mathrm{H}, H \mathrm{C}), 6.62\left(\mathrm{~s}, 2 \mathrm{H}, \mathrm{H}_{2} \mathrm{~N} \mathrm{D} \mathrm{D}_{2} \mathrm{O}\right.$-exchangeable), $7.28(\mathrm{~m}, 5 \mathrm{H}, H \mathrm{C}$ aromatic), 7.98( $\mathrm{d}, 1 \mathrm{H}, H \mathrm{C}, J=7.2 \mathrm{~Hz})$, $8.58(\mathrm{~d}, 1 \mathrm{H}, H \mathrm{C}$ aromatic, $J=7.5 \mathrm{~Hz}), 12.5(\mathrm{~s}, 1 \mathrm{H}, H \mathrm{O}$ acid, $\mathrm{D}_{2} \mathrm{O}$-exchangeable), ${ }^{13} \mathrm{C}$ NMR (DMSO- $d_{6}$ ): $\delta 30.2$ $(\mathrm{CH}), 60.2(\mathrm{CH}) 118(\mathrm{C} \equiv \mathrm{N}), 128.2(\mathrm{CH}), 129.6(\mathrm{CH})$, $133.5(\mathrm{CH}), 136.55(\mathrm{CH}), 138.6(\mathrm{CH}), 157.2(\mathrm{CH}), 166(C$ $=\mathrm{O}), 176(\mathrm{CH}-\mathrm{O}) \mathrm{MS}\left(\mathrm{m} / \mathrm{z}\right.$, aband.\%): 293( $\left.\mathrm{M}^{+}, 100 \%\right)$, 216(44.21\%), 184 (36.2\%).

\section{2-Amino-3-(Ethoxycarbonyl)-4-(P-Tolyl)-4H-} Pyrano[2,3-c]Pyridine-8-Carboxylic Acid (4c):

$\mathrm{C}_{19} \mathrm{H}_{18} \mathrm{~N}_{2} \mathrm{O}_{5}(354.12)$, brown $\left(233-235^{\circ} \mathrm{C}\right)$, Elemental analysis: C: 64.40(64.38), H: 5.12(5.14), N: 7.91(7.92), IR $(\mathrm{KBr}) \quad \bar{V}_{\max } / \mathrm{cm}^{-1}: \quad 3510(\mathrm{OH}), \quad 3325-3210\left(\mathrm{NH}_{2}\right)$ $1715(\mathrm{C}=\mathrm{O}) .{ }^{1} \mathrm{HNMR}\left(\mathrm{DMSO}-d_{6}\right): \delta 1.22\left(\mathrm{t}, 3 \mathrm{H}, H_{3} \mathrm{C}, J\right.$ $=3.1 \mathrm{~Hz}), 2.19\left(\mathrm{~s}, 3 \mathrm{H}, \mathrm{H}_{3} \mathrm{C}\right), 3.98\left(\mathrm{q}, 2 \mathrm{H}, \mathrm{H}_{2} \mathrm{C} \mathrm{J}=3.1 \mathrm{~Hz}\right)$, 4.65(s, $1 \mathrm{H}, H \mathrm{C}), 6.81\left(\mathrm{~s}, 2 \mathrm{H}, \mathrm{H}_{2} \mathrm{~N} \mathrm{D}_{2} \mathrm{O}\right.$-exchangeable), 7.1-7.38(m, 4H, HC aromatic), 8.05(d, 1H, HC, $J=$ $12 \mathrm{~Hz}), 8.58(\mathrm{~d}, 1 \mathrm{H}, H \mathrm{C}$ aromatic, $J=12 \mathrm{~Hz}), 12.51(\mathrm{~s}, 1 \mathrm{H}$, $\mathrm{HO}$ acid, $\mathrm{D}_{2} \mathrm{O}$-exchangeable), ${ }^{13} \mathrm{C}$ NMR (DMSO- $\left.d_{6}\right): \delta$ 15.2 $\left(\mathrm{CH}_{3}\right), 21.2\left(\mathrm{CH}_{3}\right), 42.3(\mathrm{CH}), 60.2(\mathrm{CH}) 78.5(\mathrm{CH})$, $125.6(\mathrm{CH}), \quad 128.2(\mathrm{CH}), \quad 129.6(\mathrm{CH}), \quad 133.5(\mathrm{CH})$, $136.55(\mathrm{CH}), 138.6(\mathrm{CH}), 159.5(\mathrm{CH}), 168(\mathrm{C}=\mathrm{O}), 170(\mathrm{C}$ = O) $\mathrm{MS}(\mathrm{m} / \mathrm{z}$, aband. $\%): 354\left(\mathrm{M}^{+}, 100 \%\right), 281(12.3 \%)$, $275(52.3 \%), 91(14.3 \%)$.

\section{2-Amino-3-Cyano-4-(P-Tolyl)-4H-Pyrano[2,3- c]Pyridine-8-Carboxylic Acid (4d)}

$\mathrm{C}_{17} \mathrm{H}_{13} \mathrm{~N}_{3} \mathrm{O}_{3}$ (307.31), Reddish brown $\left(245-247^{\circ} \mathrm{C}\right)$, Elemental analysis: C: 66.44(66.43), H: 4.26(4.28), N: 13.67(13.69), IR (KBr) $\bar{V}_{\max } / \mathrm{cm}^{-1}: 3489(\mathrm{OH}), 3310-$ $3250\left(\mathrm{NH}_{2}\right) 2230(\mathrm{C} \equiv \mathrm{N}) .{ }^{1} \mathrm{HNMR}\left(\mathrm{DMSO}-\mathrm{d}_{6}\right): \delta 2.3(\mathrm{~s}$, $\left.3 \mathrm{H}, \mathrm{H}_{3} \mathrm{C}\right) \quad 4.51(\mathrm{~s}, 1 \mathrm{H}, H \mathrm{C}), \quad 6.62\left(\mathrm{~s}, 2 \mathrm{H}, \mathrm{H}_{2} \mathrm{~N} \quad \mathrm{D}_{2} \mathrm{O}\right.$ exchangeable), 7.1-7.38(m, $4 \mathrm{H}, H \mathrm{C}$ aromatic $), 7.98(\mathrm{~d}$, $1 \mathrm{H}, H \mathrm{C}, J=7.2 \mathrm{~Hz}), 8.823(\mathrm{~d}, 1 \mathrm{H}, H \mathrm{C}$ aromatic, $J=$ $7.5 \mathrm{~Hz}), 12.02\left(\mathrm{~s}, 1 \mathrm{H}, \mathrm{HO}\right.$ acid, $\mathrm{D}_{2} \mathrm{O}$ - exchangeable $),{ }^{13} \mathrm{C}$ NMR $\left(\mathrm{DMSO}-d_{6}\right): \delta 21.2\left(\mathrm{CH}_{3}\right), 30.2(\mathrm{CH}), 60.2(\mathrm{CH})$ 
$118(C \mathrm{~N}), \quad 128.2(\mathrm{CH}), \quad 129.6(\mathrm{CH}), \quad 133.5(\mathrm{CH})$, $136.55(\mathrm{CH}), \quad 138.6(\mathrm{CH}), \quad 157.2(\mathrm{CH}), \quad 166(\mathrm{C}=\mathrm{O})$, $176(\mathrm{CH}-\mathrm{O})$ MS (m/z, aband.\%): 307( $\left.\mathrm{M}^{+}, \quad 100 \%\right)$, 203(38.4\%), 241(10.3\%).

\section{2-Amino-4-(4-Chlorophenyl)-3-(Ethoxycarbonyl)-} 4H-Pyrano[2,3-c]Pyridine-8-Carboxylic Acid (4e)

$\mathrm{C}_{18} \mathrm{H}_{15} \mathrm{ClN}_{2} \mathrm{O}_{5}$ (374.07), red $\left(287-289^{\circ} \mathrm{C}\right)$, Elemental analysis: C: 57.69(57.67), H: 4.03(4.07), N: 7.47(7.45), Cl: 9.49 (9.51); IR (KBr) $\bar{V}_{\max } / \mathrm{cm}^{-1}: 3523(\mathrm{OH}), 3423-$ 3352 $\left(\mathrm{NH}_{2}\right), 1702(\mathrm{C}=\mathrm{O}) .{ }^{1} \mathrm{HNMR}\left(\mathrm{DMSO}-d_{6}\right): \delta 1.15(\mathrm{t}$, $\left.3 \mathrm{H}, H_{3} \mathrm{C}, J=3.1 \mathrm{~Hz}\right), 4.02\left(\mathrm{q}, 2 \mathrm{H}, H_{2} \mathrm{C}, J=3.1 \mathrm{~Hz}\right)$, 4.81(s, $1 \mathrm{H}, H \mathrm{C}), 6.85\left(\mathrm{~s}, 2 \mathrm{H}, \mathrm{H}_{2} \mathrm{~N} \mathrm{D}_{2} \mathrm{O}\right.$-exchangeable), 7.38$7.42(\mathrm{~m}, 4 \mathrm{H}, H \mathrm{C}$ aromatic), $8.10(\mathrm{~d}, 1 \mathrm{H}, H \mathrm{C}, J=12 \mathrm{~Hz})$, $8.58(\mathrm{~d}, 1 \mathrm{H}, H \mathrm{C}$ aromatic, $J=12 \mathrm{~Hz}), 12.55(\mathrm{~s}, 1 \mathrm{H}, H \mathrm{O}$ acid, $\mathrm{D}_{2} \mathrm{O}$-exchangeable), ${ }^{13} \mathrm{C}$ NMR (DMSO- $\left.d_{6}\right): \delta 14.2\left(\mathrm{CH}_{3}\right)$, $45.2(\mathrm{CH}), 62.3\left(\mathrm{CH}_{2}\right), 79.2(\mathrm{CH}), 125.8(\mathrm{CH}), 133.8(\mathrm{CH})$, 135.5(CH), $138.55(\mathrm{CH}), \quad 138.6(\mathrm{CH}), \quad 160.2(\mathrm{CH})$,

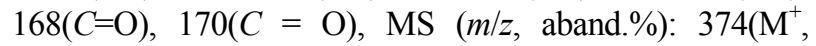
$100 \%), 301(12.5 \%), 272(35.62 \%), 111.2(18.2 \%)$.

\section{2-Amino-4-(4-Chlorophenyl)-3-Cyano-4H-}

Pyrano[2,3-c]Pyridine-8-Carboxylic Acid (4f)

$\mathrm{C}_{16} \mathrm{H}_{10} \mathrm{ClN}_{3} \mathrm{O}_{3} \quad(327.72), \quad$ red $\quad\left(275-277^{\circ} \mathrm{C}\right)$, Elemental analysis: C: 58.64(58.65), H: 3.08(3.10), N: 12.82(12.1), Cl: 10.82(10.80), IR (KBr) $\bar{V} \max / \mathrm{cm}^{-1}$ : 3455(OH), 3320-3150(NH2) 2254(C $\equiv \mathrm{N}) .{ }^{1} \mathrm{HNMR}$ (DMSO- $\left.d_{6}\right): \delta 4.86(\mathrm{~s}, 1 \mathrm{H}, H \mathrm{C}), 6.54\left(\mathrm{~s}, 2 \mathrm{H}, \mathrm{H}_{2} \mathrm{~N} \mathrm{D} \mathrm{D}_{2} \mathrm{O}-\right.$ exchangeable), 7.22-7.38(m, 4H, HC aromatic), $8.02(\mathrm{~d}, 1 \mathrm{H}, H \mathrm{C}, J=7.5 \mathrm{~Hz}), 8.85(\mathrm{~d}, 1 \mathrm{H}, H \mathrm{C}$ aromatic, $J=7.5 \mathrm{~Hz}), 12.5\left(\mathrm{~s}, 1 \mathrm{H}, \mathrm{HO}\right.$ acid, $\mathrm{D}_{2} \mathrm{O}$-exchangeable $)$, ${ }^{13} \mathrm{C}$ NMR (DMSO- $\left.d_{6}\right): \delta 28.2(\mathrm{CH}), 61.2(\mathrm{CH}), 118(\mathrm{C}$ $\equiv \mathrm{N}), \quad 124.5(\mathrm{CH}), \quad 131.2 \quad(\mathrm{CH}), \quad 133.5 \quad(\mathrm{CH})$, $136.55(\mathrm{CH}), 138.6(\mathrm{CH}), 157.2(\mathrm{CH}), 166(\mathrm{C}=\mathrm{O})$, 176(CH-O) MS (m/z, aband.\%): 327(M+, 100\%), 216(40.2\%), 138(50.01\%).

\section{2-Amino-4-(4-Bromophenyl)-3-(Ethoxycarbonyl)-} 4H-Pyrano[2,3-c]Pyridine-8-Carboxylic Acid (4g)

$\mathrm{C}_{18} \mathrm{H}_{15} \mathrm{BrN}_{2} \mathrm{O}_{5}$ (418.02), Reddish yellow (290$292^{\circ} \mathrm{C}$ ), Elemental analysis: $\mathrm{C}$ : 51.57(51.58), $\mathrm{H}$ : 3.61(3.63), N: 6.68(6.70), Br: 19.06(19.08), IR (KBr) $\bar{V}{ }_{\max } / \mathrm{cm}^{-1}: 3523(\mathrm{OH}), 3423-3352\left(\mathrm{NH}_{2}\right), 1702(\mathrm{C}=$ O). ${ }^{1} \mathrm{HNMR}\left(\mathrm{DMSO}-d_{6}\right): \delta \delta 1.021\left(\mathrm{t}, 3 \mathrm{H}, \mathrm{H}_{3} \mathrm{C}, \mathrm{J}=\right.$ $3.1 \mathrm{~Hz}), 4.02\left(\mathrm{q}, 2 \mathrm{H}, \mathrm{H}_{2} \mathrm{C}, J=3.1 \mathrm{~Hz}\right), 4.81(\mathrm{~s}, 1 \mathrm{H}, H \mathrm{C})$, 6.77(s, $2 \mathrm{H}, \mathrm{H}_{2} \mathrm{~N} \mathrm{D}_{2} \mathrm{O}$-exchangeable), 7.38-7.62(m, $4 \mathrm{H}, H \mathrm{C}$ aromatic), $8.12(\mathrm{~d}, 1 \mathrm{H}, H \mathrm{C}, J=12 \mathrm{~Hz})$, $8.58(\mathrm{~d}, 1 \mathrm{H}, H \mathrm{C}$ aromatic, $J=12 \mathrm{~Hz}), 12.55(\mathrm{~s}, 1 \mathrm{H}, H \mathrm{O}$ acid, $\mathrm{D}_{2}$ Oexchangeable), ${ }^{13} \mathrm{C}$ NMR (DMSO-d $): \delta 14.5$ $\left(\mathrm{CH}_{3}\right), 42.3(\mathrm{CH}), 61.9\left(\mathrm{CH}_{2}\right), 77.9(\mathrm{CH}), 118.2(\mathrm{CH})$, $131.2(\mathrm{CH}), \quad 133.2(\mathrm{CH}), \quad 136.2(\mathrm{CH}), 138.6(\mathrm{CH})$, $157.5(C \mathrm{H}), 168.6(C=\mathrm{O}), 170.1(C=\mathrm{O}) \mathrm{MS}(\mathrm{m} / \mathrm{z}$, aband.\%): 418( $\left.\mathrm{M}^{+}, 100 \%\right), 202(38.2 \%), 185(23.6 \%)$.
2-Amino-4-(4-Bromophenyl)-3-Cyano-4H-

Pyrano[2,3-c]Pyridine-8-Carboxylic Acid (4h)

$\mathrm{C}_{16} \mathrm{H}_{10} \mathrm{BrN}_{3} \mathrm{O}_{3} \quad(372.18), \quad$ Yellow $\quad\left(288-289^{\circ} \mathrm{C}\right)$, Elemental analysis: $\mathrm{C}$ : 51.64(51.66), $\mathrm{H}: 2.71(2.72), \mathrm{N}$ : 11.29(11.30), Br: 21.47(21.49), IR (KBr) $\bar{V}_{\max } / \mathrm{cm}^{-1}$ : $3455(\mathrm{OH}), \quad 3320-3150\left(\mathrm{NH}_{2}\right) \quad 2254(\mathrm{C} \equiv \mathrm{N}) . \quad{ }^{1} \mathrm{HNMR}$ $\left(\mathrm{DMSO}-d_{6}\right): \delta 4.72(\mathrm{~s}, 1 \mathrm{H}, H \mathrm{C}), 6.61\left(\mathrm{~s}, 1 \mathrm{H}, \mathrm{H}_{2} \mathrm{~N} \mathrm{D} \mathrm{D}_{2} \mathrm{O}\right.$ exchangeable), 7.28-7.44(m, $4 \mathrm{H}, H \mathrm{C}$ aromatic $), 8.05(\mathrm{~d}, 1$ $\mathrm{H}, H \mathrm{C}, J=7.5 \mathrm{~Hz}), 8.61(\mathrm{~d}, 1 \mathrm{H}, H \mathrm{C}$ aromatic, $J=7.5 \mathrm{~Hz})$, $12.5\left(\mathrm{~s}, 1 \mathrm{H}, \quad \mathrm{HO}\right.$ acid, $\mathrm{D}_{2} \mathrm{O}$-exchangeable $),{ }^{13} \mathrm{C} \quad \mathrm{NMR}$ (DMSO-d $\left.)_{6}\right): \delta 29.3\left(\mathrm{CH}_{2}\right), 60.2(\mathrm{CH}), 118.1(\mathrm{C} \equiv \mathrm{N}), 120.3$ $(\mathrm{CH}), 131.1(\mathrm{CH}), 134.2(\mathrm{CH}), 136.2(\mathrm{CH}), 138.6(\mathrm{CH})$, $158.2(\mathrm{CH}), 168(\mathrm{C}=\mathrm{O}), 176.9(\mathrm{C}-\mathrm{O})$, MS $(\mathrm{m} / \mathrm{z}$, aband.\%): 372( $\left.\mathrm{M}^{+}, 100 \%\right), 218(65.3 \%), 154(22.5 \%)$.

2-Amino-3-(Ethoxycarbonyl)-4-(4-Methoxyphenyl)4H-Pyrano[2,3-c]Pyridine-8-Carboxylic Acid (4i)

$\mathrm{C}_{19} \mathrm{H}_{18} \mathrm{~N}_{2} \mathrm{O}_{6}$ (370.12), Reddish brown $\left(255-257^{\circ} \mathrm{C}\right)$, Elemental analysis: C: 61.62 (61.60), H: 4.90 (4.88), N: 7.56(7.58), IR (KBr) $\bar{V}_{\max } / \mathrm{cm}^{-1}: 3455(\mathrm{OH}), 3325-$ $3210\left(\mathrm{NH}_{2}\right)$ 1705(C = O). ${ }^{1} \mathrm{HNMR}\left(\mathrm{DMSO}-d_{6}\right): \delta 1.22(\mathrm{t}$, $\left.3 \mathrm{H}, \mathrm{H}_{3} \mathrm{C}, J=3.1 \mathrm{~Hz}\right), 3.79\left(\mathrm{q}, 3 \mathrm{H}, \mathrm{H}_{3} \mathrm{CO}, J=3.1 \mathrm{~Hz}\right.$ ), $3.98\left(\mathrm{~s}, 2 \mathrm{H}, \mathrm{H}_{2} \mathrm{C}\right), 4.74(\mathrm{~s}, 1 \mathrm{H}, H \mathrm{C}), 6.81\left(\mathrm{~s}, 1 \mathrm{H}, H_{2} \mathrm{~N} \mathrm{D}_{2} \mathrm{O}\right.$ exchangeable), 6.88-7.39 (m, $4 \mathrm{H}, H \mathrm{C}$ aromatic), 8.05(d, $1 \mathrm{H}, H \mathrm{C}, J=7.2 \mathrm{~Hz}), 8.58(\mathrm{~d}, 1 \mathrm{H}, H \mathrm{C}$ aromatic, $J=$ 7.2Hz), 12.54(s, $1 \mathrm{H}, \mathrm{HO}$ acid, $\mathrm{D}_{2} \mathrm{O}$-exchangeable), ${ }^{13} \mathrm{C}$ NMR (DMSO- $\left.d_{6}\right): \delta 14.2\left(\mathrm{CH}_{3}\right), 56.2\left(\mathrm{OCH}_{3}\right), 42.3$ $(\mathrm{CH}), 62.3\left(\mathrm{CH}_{2}\right), 78.5(\mathrm{CH}), 115.2(\mathrm{CH}), 131.2(\mathrm{CH})$, $133.2(\mathrm{CH}), 136.2(\mathrm{CH}), 138.6(\mathrm{CH}), 159.5(\mathrm{CH}), 168$ $(C=\mathrm{O}), 170(C=\mathrm{O}) \mathrm{MS}\left(\mathrm{m} / \mathrm{z}\right.$, aband.\%): $370\left(\mathrm{M}^{+}\right.$, 100\%), 202(25.3\%), 136(4.2\%), 73 (14.3\%).

\section{2-Amino-3-Cyano-4-(4-Methoxyphenyl)-4H-}

Pyrano[2,3-c] Pyridine-8-Carboxylic Acid (4j)

$\mathrm{C}_{17} \mathrm{H}_{13} \mathrm{~N}_{3} \mathrm{O}_{3}$ (323.31), Brown $\left(2249-250^{\circ} \mathrm{C}\right)$, Elemental analysis: $\mathrm{C}: 63.16(63.18), \mathrm{H}: 4.05(4.03), \mathrm{N}: 13.00(13.01)$, IR (KBr) $\quad \bar{V}_{\max } / \mathrm{cm}^{-1}: 3488(\mathrm{OH}), \quad 3310-3273\left(\mathrm{NH}_{2}\right)$ $2230(\mathrm{C} \equiv \mathrm{N}) .{ }^{1} \mathrm{HNMR}\left(\mathrm{DMSO}-d_{6}\right): \delta 3.41\left(\mathrm{~s}, 3 \mathrm{H}, \mathrm{H}_{3} \mathrm{CO}\right)$, 4.63(s,1H, HC), 6.72(s, $1 \mathrm{H}, H_{2} \mathrm{~N} \mathrm{D}_{2} \mathrm{O}$-exchangeable), $6.88-7.03(\mathrm{~m}, 4 \mathrm{H}, H \mathrm{C}$ aromatic $), 8.00(\mathrm{~d}, 1 \mathrm{H}, H \mathrm{C}, J=$ $7.5 \mathrm{~Hz}), 8.61(\mathrm{~d}, 1 \mathrm{H}, H \mathrm{C}$ aromatic, $J=7.5 \mathrm{~Hz}), 12.5(\mathrm{~s}, 1 \mathrm{H}$, $\mathrm{HO}$ acid, $\mathrm{D}_{2} \mathrm{O}$-exchangeable), ${ }^{13} \mathrm{C}$ NMR (DMSO- $d_{6}$ ): $\delta$ $30.2(\mathrm{CH}), 58.2\left(\mathrm{OCH}_{3}\right), 60.2(\mathrm{CH}) 119.2(\mathrm{CN}), 115.6$ $(\mathrm{CH}), 129.6(\mathrm{CH}), 133.5(\mathrm{CH}), 138.55(\mathrm{CH}), 157.2(C-\mathrm{O})$, $166(\mathrm{C}=\mathrm{O}), 176(\mathrm{CH}-\mathrm{O}), \mathrm{MS}\left(\mathrm{m} / \mathrm{z}\right.$, aband.\%): 323( $\mathrm{M}^{+}$, $100 \%), 216(50.8 \%), 107(25.3 \%)$.

\section{2-Amino-3-(Ethoxycarbonyl)-4-(4-Nitrophenyl)-} 4H-Pyrano[2,3-c]Pyridine-8-Carboxylic Acid (4k)

$\mathrm{C}_{18} \mathrm{H}_{15} \mathrm{~N}_{3} \mathrm{O}_{7}$ (385.09), yellow $\left(291-292^{\circ} \mathrm{C}\right)$, Elemental analysis: $\mathrm{C}: 56.11 \quad(56.13), \quad \mathrm{H}: 3.92 \quad(3.90), \quad \mathrm{N}$ : 10.91(10.93), IR (KBr) $\bar{V}{ }_{\max } / \mathrm{cm}^{-1}: 3510(\mathrm{OH}), 3310-$ 
3150 $\left(\mathrm{NH}_{2}\right)$ 1699(C=O). ${ }^{1} \mathrm{HNMR}\left(\mathrm{DMSO}-d_{6}\right): \delta 1.022$ (t, $\left.3 \mathrm{H}, H_{3} \mathrm{C}, J=3.1 \mathrm{~Hz}\right), 3.98\left(\mathrm{q}, 2 \mathrm{H}, H_{2} \mathrm{C}, J=3.1 \mathrm{~Hz}\right)$, 4.74(s, $1 \mathrm{H}, H \mathrm{C}), 6.81\left(\mathrm{~s}, 2 \mathrm{H}, \mathrm{H}_{2} \mathrm{~N} \mathrm{D}_{2} \mathrm{O}\right.$-exchangeable), 7.52-8.00 (m, 4H, HC aromatic), 8.05(d, 1H, HC, J = $7.2 \mathrm{~Hz}), 8.58(\mathrm{~d}, 1 \mathrm{H}, \mathrm{HC}$ aromatic, $\mathrm{J}=7.2 \mathrm{~Hz}), 12.56(\mathrm{~s}$, $1 \mathrm{H}, \mathrm{HO}$ acid, $\mathrm{D}_{2} \mathrm{O}$-exchangeable), ${ }^{13} \mathrm{C}$ NMR (DMSO$\left.\mathrm{d}_{6}\right): \delta 14.2\left(\mathrm{CH}_{3}\right), 42.3(\mathrm{CH}), 62.3\left(\mathrm{CH}_{2}\right), 78.5(\mathrm{CH})$, $124.2(\mathrm{CH}), 129.3(\mathrm{CH}), 133.2(\mathrm{CH}), 136.2(\mathrm{CH}), 138.6$ $(\mathrm{CH}), 159.5(\mathrm{CH}), 168(\mathrm{C}=\mathrm{O}), 170(\mathrm{C}=\mathrm{O}) \mathrm{MS}(\mathrm{m} / \mathrm{z}$, aband.\%): $385\left(\mathrm{M}^{+}, 100 \%\right), 202(33.3 \%), 180(21.5 \%)$.

\section{2-Amino-3-Cyano-4-(4-Nitrophenyl)-4H-}

Pyrano[2,3-c]Pyridine-8-Carboxylic Acid (4l)

$\mathrm{C}_{16} \mathrm{H}_{10} \mathrm{~N}_{4} \mathrm{O}_{5}$ (338.07), Dark yellow $\left(281-283^{\circ} \mathrm{C}\right)$, Elemental analysis: C: 56.81(56.83), H: 2.98(2.96), N: 16.56(16.55), IR (KBr) $\bar{V}_{\max } / \mathrm{cm}^{-1}: 3510(\mathrm{OH}), 3320-$ 3200 $\left(\mathrm{NH}_{2}\right)$ 2243(C $\left.\equiv \mathrm{N}\right) .{ }^{1} \mathrm{HNMR}\left(\mathrm{DMSO}-d_{6}\right): \delta 4.70(\mathrm{~s}$,

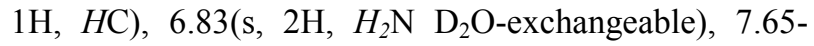
7.96(m, 4H, HC aromatic), 8.05(d, $1 \mathrm{H}, H \mathrm{C}, J=12 \mathrm{~Hz})$, $8.57(\mathrm{~d}, 1 \mathrm{H}, H \mathrm{C}$ aromatic, $J=12 \mathrm{~Hz}), 12.5(\mathrm{~s}, 1 \mathrm{H}, H \mathrm{O}$ acid, $\mathrm{D}_{2} \mathrm{O}$-exchangeable), ${ }^{13} \mathrm{C} \quad \mathrm{NMR} \quad\left(\mathrm{DMSO}-d_{6}\right): \quad \delta \quad 28.7$ $\left(\mathrm{CH}_{2}\right), 58.6(\mathrm{CH}), 118.1(\mathrm{C} \equiv \mathrm{N}), 124.3(\mathrm{CH}), 124.1(\mathrm{CH})$, $128.9(\mathrm{CH}), \quad 133.2(\mathrm{CH}), \quad 136.2(\mathrm{CH}), \quad 138.6(\mathrm{CH})$, $142.1(\mathrm{CH}), \quad 145.1(\mathrm{CH}), \quad 150.2(\mathrm{CH}), \quad 158(\mathrm{CH})$, 167.9 $(C=\mathrm{O}), \quad 177(C-\mathrm{O})$, MS (m/z,aband.\%): 338 $\left(\mathrm{M}^{+}\right.$, 100\%), 241(44.3\%), 122(36.2\%).

\section{2-Amino-3-(Ethoxycarbonyl)-4-(Furan-2-yl)-4H- Pyrano[2,3-c]Pyridine-8-Carboxylic Acid (4m)}

$\mathrm{C}_{16} \mathrm{H}_{14} \mathrm{~N}_{2} \mathrm{O}_{6}(330.09)$, brown $\left(244-245^{\circ} \mathrm{C}\right)$, Elemental analysis: C: 58.18 (58.19), H: 4.27 (4.29), N: 8.48(8.46), IR $(\mathrm{KBr}) \quad \bar{V}_{\max } / \mathrm{cm}^{-1}: \quad 3530(\mathrm{OH}), \quad 3320-3225\left(\mathrm{NH}_{2}\right)$ 1699(C = O). ${ }^{1} \mathrm{HNMR}\left(\mathrm{DMSO}-d_{6}\right): \delta 1.22\left(\mathrm{t}, 3 \mathrm{H}, H_{3} \mathrm{C}, J\right.$ $=3.1 \mathrm{~Hz}), 3.98\left(\mathrm{q}, 2 \mathrm{H}, \mathrm{H}_{2} \mathrm{C}, J=3.1 \mathrm{~Hz}\right), 4.82(\mathrm{~s}, 2 \mathrm{H}, H \mathrm{C})$, $6.22(\mathrm{~d}, 1 \mathrm{H}, H \mathrm{C}$ furan, $J=1.2 \mathrm{~Hz}), 6.51(\mathrm{t}, 1 \mathrm{H}, H \mathrm{C}$ furan, $J=3.1 \mathrm{~Hz}), 6.81\left(\mathrm{~s}, 1 \mathrm{H}, \mathrm{H}_{2} \mathrm{~N} \mathrm{D}_{2} \mathrm{O}\right.$ exchangeable), 7.50 (d, $1 \mathrm{H}, H \mathrm{C}$ furan, $J=1.2 \mathrm{~Hz}), 8.05(\mathrm{~d}, 1 \mathrm{H}, \underline{H C}, J=7.2 \mathrm{~Hz})$, $8.58(\mathrm{~d}, 1 \mathrm{H}, H \mathrm{C}$ aromatic, $J=7.2 \mathrm{~Hz}), 12.56(\mathrm{~s}, 1 \mathrm{H}, H \mathrm{O}$ acid, $\mathrm{D}_{2} \mathrm{O}$ exchangeable), ${ }^{13} \mathrm{C}$ NMR (DMSO- $\left.d_{6}\right): \delta 14.2$ $\left(\mathrm{CH}_{3}\right), 32.2(\mathrm{CH}), 62.3\left(\mathrm{CH}_{2}\right), 79.1(\mathrm{CH}), 107(\mathrm{CH}), 110$ $(\mathrm{CH}), 133.2(\mathrm{CH}), 136.2(\mathrm{CH}), 138.6(\mathrm{CH}), 157.5(\mathrm{CH})$,

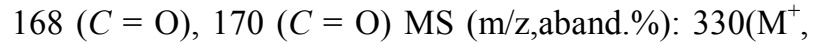
$100 \%), 288(12.3 \%), 257(48.2 \%)$.

\section{2-Amino-3-Cyano-4-(Furan-2-yl)-4H-Pyrano[2,3- c]Pyridine-8-Carboxylic Acid (4n)}

$\mathrm{C}_{14} \mathrm{H}_{9} \mathrm{~N}_{3} \mathrm{O}_{3}(283.06)$, brown $\left(232-234^{\circ} \mathrm{C}\right)$, Elemental analysis: C: 59.37(59.40), H: 3.20(3.18), N: 14.84(14.86), IR (KBr) $\bar{V}_{\max } / \mathrm{cm}^{-1}: 3501(\mathrm{OH}), 3352-3268\left(\mathrm{NH}_{2}\right) 2249(\mathrm{C}$ $\equiv \mathrm{N}) .{ }^{1} \mathrm{HNMR}\left(\mathrm{DMSO}_{6}\right): \delta 4.87(\mathrm{~s}, 1 \mathrm{H}, H \mathrm{C}), 5.98(\mathrm{~d}, 1 \mathrm{H}$, $H \mathrm{C}$ furan, $J=1.2 \mathrm{~Hz}), 6.09(\mathrm{t}, 1 \mathrm{H}, H \mathrm{C}$ furan,$J=1.2 \mathrm{~Hz}$ ), 6.72(s, $2 \mathrm{H}, \mathrm{H}_{2} \mathrm{~N} \mathrm{D}_{2} \mathrm{O}$-exchangeable), 7.51(d, $1 \mathrm{H}, H \mathrm{C}$ furan, $J=1.2 \mathrm{~Hz}), 8.00(\mathrm{~d}, 1 \mathrm{H}, H \mathrm{C}, J=7.5 \mathrm{~Hz}), 8.61(\mathrm{~d}, 1 \mathrm{H}$, $H \mathrm{C}$ aromatic, $J=7.5 \mathrm{~Hz}), 12.5\left(\mathrm{~s}, 1 \mathrm{H}, \mathrm{HO}\right.$ acid, $\mathrm{D}_{2} \mathrm{O}$ exchangeable), ${ }^{13} \mathrm{C}$ NMR (DMSO- $\left.d_{6}\right): \delta 30.2(\mathrm{CH})$, $60.2(\mathrm{CH}), 107.3(\mathrm{CH}), 110.6(C \mathrm{H}), 119.2(\mathrm{C} \equiv \mathrm{N})$, $129.6(\mathrm{CH}), 133.5(\mathrm{CH}), 142.5(\mathrm{CH}), 157.2(C-\mathrm{O}), 166$ $(C=\mathrm{O}), 176(\mathrm{CH}-\mathrm{O}) \mathrm{MS}\left(\mathrm{m} / \mathrm{z}\right.$, aband.\%): $283\left(\mathrm{M}^{+}\right.$, 100\%), 216 (48.3\%), 67 (12.3\%).

2-Amino-3-(Ethoxycarbonyl)-4-(Pyridin-4-yl)-4HPyrano[2,3-c]Pyridine-8-Carboxylic acid (4o)

$\mathrm{C}_{17} \mathrm{H}_{15} \mathrm{~N}_{3} \mathrm{O}_{5}(341.10)$, orange $\left(273-275^{\circ} \mathrm{C}\right)$, Elemental analysis: $\quad \mathrm{C}: \quad 59.82 \quad(59.80), \quad \mathrm{H}: \quad 4.43(4.45), \quad \mathrm{N}$ : 12.31(12.32), IR (KBr) $\bar{V}_{\max } / \mathrm{cm}^{-1}: 3530(\mathrm{OH}), 3320-$ 3225 $\left(\mathrm{NH}_{2}\right) 1699(\mathrm{C}=\mathrm{O}) .{ }^{1} \mathrm{HNMR}\left(\mathrm{DMSO}-d_{6}\right): \delta 1.15(\mathrm{t}$, $\left.3 \mathrm{H}, H_{3} \mathrm{C}, J=3.1 \mathrm{~Hz}\right), 3.98\left(\mathrm{q}, 2 \mathrm{H}, \mathrm{H}_{2} \mathrm{C}, J=3.1 \mathrm{~Hz}\right)$, 4.72(s, $1 \mathrm{H}, H \mathrm{C}), 6.83\left(\mathrm{~s}, 1 \mathrm{H}, \mathrm{H}_{2} \mathrm{~N} \mathrm{D} \mathrm{D}_{2} \mathrm{O}\right.$-exchangeable), $7.25(\mathrm{~d}, 2 \mathrm{H}, H \mathrm{C}$ pyridine, $J=1.2 \mathrm{~Hz}), 8.05(\mathrm{~d}, 1 \mathrm{H}, H \mathrm{C}, J$ $=7.2 \mathrm{~Hz}), 8.40(\mathrm{~d}, 2 \mathrm{H}, H \mathrm{C}$ pyridine, $J=1.2 \mathrm{~Hz}), 8.58(\mathrm{~d}$, $1 \mathrm{H}, H \mathrm{C}$ aromatic, $J=7.2 \mathrm{~Hz}), 12.56\left(\mathrm{~s}, 1 \mathrm{H}, \mathrm{HO}\right.$ acid, $\mathrm{D}_{2} \mathrm{O}$ exchangeable), ${ }^{13} \mathrm{C}$ NMR (DMSO- $\left.d_{6}\right): \delta 14.2\left(\mathrm{CH}_{3}\right), 42.2$ $(\mathrm{CH}), 62.3\left(\mathrm{CH}_{2}\right), 79.1(\mathrm{CH}), 124(\mathrm{CH}), 133.2(\mathrm{CH})$, $136.2(\mathrm{CH}), 138.6(\mathrm{CH}), 150.2(\mathrm{CH}), 158(\mathrm{CH}), 168(C$ $=\mathrm{O}), 170(C=\mathrm{O}), \mathrm{MS}\left(\mathrm{m} / \mathrm{z}\right.$, aband.\%): 341( $\left.\mathrm{M}^{+}, 100 \%\right)$, 288(22.3\%), 268(35.2\%).

2-Amino-3-Cyano-4-(Pyridin-4-yl)-4H-Pyrano[2,3c]Pyridine-8-Carboxylic Acid (4p)

$\mathrm{C}_{15} \mathrm{H}_{10} \mathrm{~N}_{4} \mathrm{O}_{3}$ (294.27), Dark orange $\left(256-258^{\circ} \mathrm{C}\right)$, Elemental analysis: C: 61.22(61.25), H: 3.43(3.42), N: 19.04(19.06), IR (KBr) $\bar{V}_{\max } / \mathrm{cm}^{-1}: 3513(\mathrm{OH}), 3348-$ 3312 $\left(\mathrm{NH}_{2}\right) \quad 2255(\mathrm{C} \equiv \mathrm{N}) .{ }^{1} \mathrm{HNMR} \quad\left(\mathrm{DMSO}-d_{6}\right): \delta$ 4.74(s, $1 \mathrm{H}, H \mathrm{C}), 6.80\left(\mathrm{~s}, 2 \mathrm{H}, H_{2} \mathrm{~N} \mathrm{D}_{2} \mathrm{O}\right.$-exchangeable), $7.10(\mathrm{~d}, 1 \mathrm{H}, H \mathrm{C}$ pyridine, $J=3.2 \mathrm{~Hz}), 8.03(\mathrm{~d}, 1 \mathrm{H}, H \mathrm{C}$, $J=7.5 \mathrm{~Hz}), 8.45(\mathrm{~d}, 1 \mathrm{H}, H \mathrm{C}$ pyridine, $J=3.2 \mathrm{~Hz})$, $8.71(\mathrm{~d}, 1 \mathrm{H}, H \mathrm{C}$ aromatic, $J=7.5 \mathrm{~Hz}), 12.52(\mathrm{~s}, 1 \mathrm{H}, H \mathrm{O}$ acid, $\mathrm{D}_{2} \mathrm{O}$-exchangeable), ${ }^{13} \mathrm{C}$ NMR (DMSO- $\left.d_{6}\right): \delta$ $30.2(\mathrm{CH}), 60.2(\mathrm{CH}), 119.2(\mathrm{CN}), 124.2(\mathrm{CH}), 126.2$ $(\mathrm{CH}), 133.5(\mathrm{CH}), 138.3(\mathrm{CH}), 149.5(\mathrm{CH}), 157.2(\mathrm{C}$ $\mathrm{O}), 166(\mathrm{C}=\mathrm{O}), 176(\mathrm{CH}-\mathrm{O}) \mathrm{MS}(\mathrm{m} / \mathrm{z}$, aband.\%): 294( $\left.\mathrm{M}^{+}, 100 \%\right), 216(55.6 \%), 78(13.2 \%)$.

\section{Results and Discussion}

\section{Chemistry}

Green synthesis one-pot of pyrano[2,3-c]pyridine annulated heterocyclic compound via threeconstituent condensation mixture reaction of aldehydes, ethyl cyanoacetate or malononitrile and 3hydroxy picolinic acid have been accomplished by microwave irradiation (Mady et al., 2015) and thermal heating utilizing 4-Dimethylaminopyridine (DMAP) as displayed in Scheme 1. 


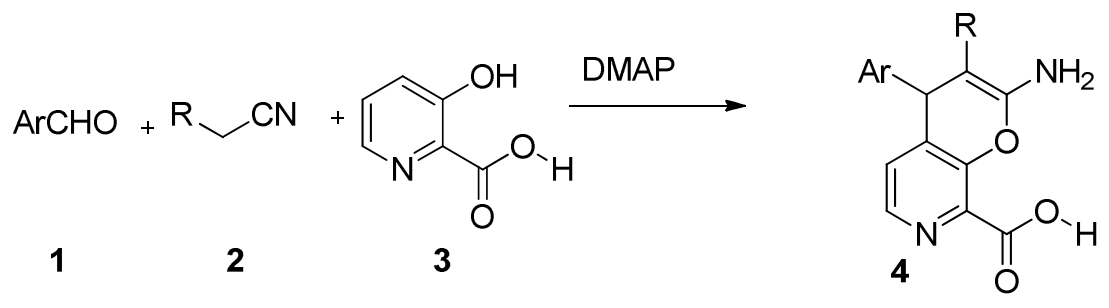

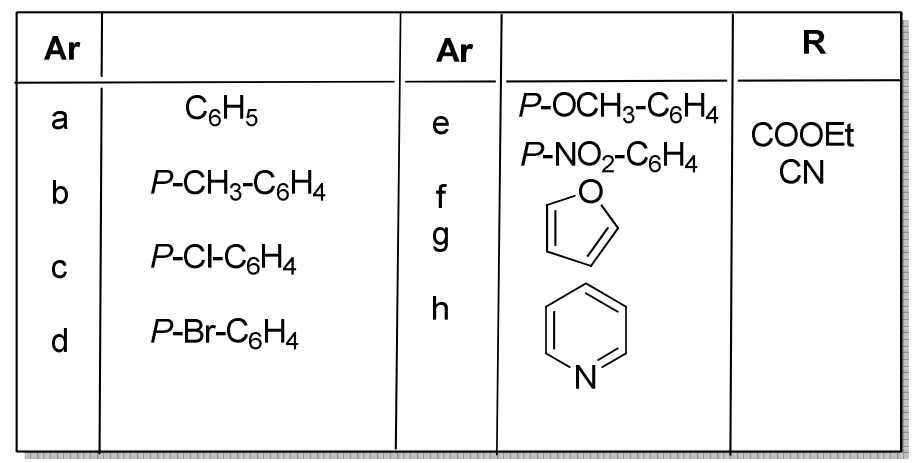

Scheme 1: Prepration of pyrano-fused heterocycles<smiles>CCOC(=O)CC#N</smiles>

Fig. 1: Synthesis of the 2-amino-3-(ethoxycarbonyl)-4-phenyl-4H-pyrano[2,3-c]pyridine-8-carboxylic acid (4a)

For this study, an intermixture of aromatic benzaldehydes $(1 \mathrm{mmol})$ and ethyl cyanoacetate or malononitrile $(1 \mathrm{mmol})$ in ethyl alcohol was preserved with DMAP $(0.1 \mathrm{mmol})$ at typical temperature. Subsequently ingesting of initial aldehyde as examined via thin layer chromatography, 3-hydroxy picolinic acid was supplementary to the response combination and reserved for magnificent further down the heat for $1 \mathrm{~min}$ in microwave instrument. Subsequently the accomplishment of the reaction examined via thin layer chromatography, the reaction combination was acquired to typical temperature and the formed precipitate was riddled off. Product $4 a$, was achieved in $85 \%$ yield in microwave irradiation, which was characterized by ${ }^{1} \mathrm{HNMR},{ }^{13} \mathrm{C}$ NMR, besides through elemental examination as displayed in Figure 1.

The optimized reaction was exhausting different accelerator for attaining the excellent concern of $4 \mathrm{a}$ are summarized in Table 1. That one was distinguished that $(20 \%)$ mol of DMAP in ethanol consequences from the greatest yield and time, the income obtained about $(71 \%)$ in thermal heating. Subsequently the reaction optimization condition was comprehensive to a variability of aromatic aldehydes with dissimilar components.

The compounds $4 \mathbf{a}-\mathbf{4 p}$ were associated with those of conventional heating and microwave irradiation. It was demonstrated high yield properties of microwave compounds than thermal performance. The microwave was high yield and in a few minutes as shown in Table 2.

The formation of pyrano[2,3-c]pyridine-8-carboxylic acid derivatives can be reorganized as tracks. Initially, the Knovengel condensation of an aldehyde and alkyl nitrile to form acrylonitrile derivative I using DMAP catalyst, which responded to produce carbonian from activated 3-hydroxypicinolic acid to give the intermediate II, which cyclized to IV in the occurrence of DMAP. As a final point, IV tautomerized to provide preferred product $\mathbf{4}$ as presented in Scheme 2 . 
Table 1: Reaction condition optimization

\begin{tabular}{llllllll}
\hline \multicolumn{2}{l}{ Catalyst Solvent } & Catalytic amount (mol \%) & MW time (min) & Yield (\%) & Heating time (h) & Yield (\%) \\
\hline 1 & Piperidine & EtOH & 20 & $5 \mathrm{~min}$ & 52 & $4 \mathrm{~h}$ & 43 \\
2 & DMAP & Neat & 20 & $5 \mathrm{~min}$ & 66 & $4 \mathrm{~h}$ & 45 \\
3 & DMAP & $\mathrm{MeOH}$ & 20 & $5 \mathrm{~min}$ & 68 & $4 \mathrm{~h}$ & 55 \\
4 & DMAP & $\mathrm{H}_{2} \mathrm{O}$ & 20 & $10 \mathrm{~min}$ & 60 & $6 \mathrm{~h}$ & 55 \\
5 & DMAP & EtOH & 10 & $5 \mathrm{~min}$ & 77 & $5 \mathrm{~h}$ & 65 \\
6 & DMAP & EtOH & 20 & $2 \mathrm{~min}$ & 85 & $4 \mathrm{~h}$ & 71 \\
7 & DMAP & EtOH & 30 & $1 \mathrm{~min}$ & 80 & $4 \mathrm{~h}$ & 68 \\
\hline
\end{tabular}

${ }^{\text {an } \text { Isolated yield }}$

Table 2: Prepration of pyrano[2,3-c]pyridine-8-carboxylic acid derivatives utilizing aromatic aldehydes, ethyl cyanoacetate or malononitrile and 3-hydroxypicinolic acid through DMAP

\begin{tabular}{|c|c|c|c|c|c|c|}
\hline \multicolumn{2}{|c|}{ Aromatic aldehydes } & \multirow{2}{*}{$\frac{\text { Product }}{\mathbf{4 a}}$} & \multirow{2}{*}{$\frac{\text { MW time }}{1 \mathrm{~min}}$} & \multirow{2}{*}{$\frac{\text { Yield a }(\%)}{85 \%}$} & \multirow{2}{*}{$\begin{array}{l}\text { Heating time } \\
\mathrm{h} h\end{array}$} & \multirow{2}{*}{$\frac{\text { Yield }(\%)}{68}$} \\
\hline 1 & $\mathrm{C}_{6} \mathrm{H}_{5}$ & & & & & \\
\hline 2 & $\mathrm{C}_{6} \mathrm{H}_{5}$ & $4 b$ & 1 min (stirring) & $77 \%$ & 30 min (stirring) & 68 \\
\hline 3 & 4- $\mathrm{CH}_{3} \mathrm{C}_{6} \mathrm{H}_{4}$ & $4 c$ & $1 \mathrm{~min}$ & $65 \%$ & $5 \mathrm{~h}$ & 52 \\
\hline 4 & 4- $\mathrm{CH}_{3} \mathrm{C}_{6} \mathrm{H}_{4}$ & 4d & 1 min (stirring) & $67 \%$ & $30 \mathrm{~min}$ (stirring) & 57 \\
\hline 5 & $4-\mathrm{ClC}_{6} \mathrm{H}_{4}$ & $4 e$ & $1 \mathrm{~min}$ & $77 \%$ & $6 \mathrm{~h}$ & 63 \\
\hline 6 & $4-\mathrm{ClC}_{6} \mathrm{H}_{4}$ & $4 f$ & 1 min (stirring) & $75 \%$ & 35 min (stirring) & 59 \\
\hline 7 & 4- $\mathrm{BrC}_{6} \mathrm{H}_{4}$ & $4 g$ & $1 \mathrm{~min}$ & $81 \%$ & $6 \mathrm{~h}$ & 65 \\
\hline 8 & 4- $\mathrm{BrC}_{6} \mathrm{H}_{4}$ & $4 \mathrm{~h}$ & 1 min (stirring) & $79 \%$ & 35 min (stirring) & 64 \\
\hline 9 & 4- $\mathrm{CH}_{3} \mathrm{OC}_{6} \mathrm{H}_{4}$ & $4 \mathbf{i}$ & $1 \mathrm{~min}$ & $63 \%$ & $5 \mathrm{~h}$ & 45 \\
\hline 10 & $4-\mathrm{CH}_{3} \mathrm{OC}_{6} \mathrm{H}_{4}$ & $4 \mathbf{j}$ & 1 min (stirring) & $67 \%$ & $30 \min$ (stirring) & 63 \\
\hline 11 & $4-\mathrm{NO}_{2} \mathrm{C}_{6} \mathrm{H}_{4}$ & $4 k$ & $1 \mathrm{~min}$ & $74 \%$ & $7 \mathrm{~h}$ & 66 \\
\hline 12 & $4-\mathrm{NO}_{2} \mathrm{C}_{6} \mathrm{H}_{4}$ & 41 & $1 \mathrm{~min}$ (stirring) & $72 \%$ & $40 \min$ (stirring) & 65 \\
\hline 13 & 2-Furanyl & $4 \mathrm{~m}$ & $1 \mathrm{~min}$ & $63 \%$ & $8 \mathrm{~h}$ & 55 \\
\hline 14 & 2-Furanyl & $4 n$ & $1 \mathrm{~min}$ (stirring) & $68 \%$ & $45 \min$ (stirring) & 57 \\
\hline 15 & Picolinaldehyde & 40 & $1 \mathrm{~min}$ & $71 \%$ & $8 \mathrm{~h}$ & 63 \\
\hline 16 & Picolinaldehyde & $4 p$ & $1 \mathrm{~min}$ (stirring) & $73 \%$ & $45 \min$ (stirring) & 59 \\
\hline
\end{tabular}

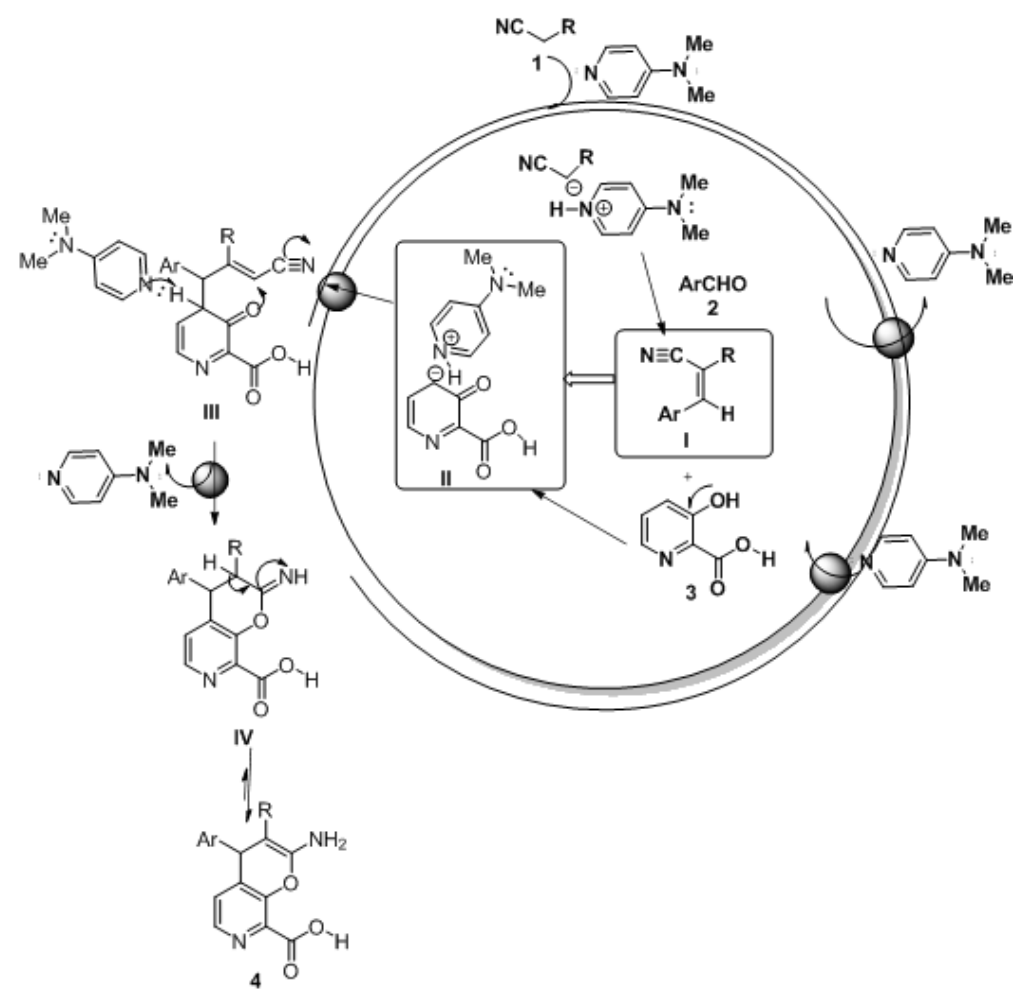

Scheme 2: Possible mechanism for development of pyrano[2,3-c]pyridine-8-carboxylic acid derivatives 


\section{Biological Activity}

\section{In vitro Cytotoxic Activity}

Antimicrobial activity was accomplished at The Regional Center for Mycology and Biotechnology (RCMB), Al-Azhar University, Cairo, Egypt. Inhibition zones of bacterial evolution premeditated for the manufactured products and standard drugs utilizing Hole-plate dispersal procedure. Six intermediate $(1 \mathrm{~cm}$ diameter) holes were finished consuming sterile cork borer in (MHA) Mullere Hinton agar sterile plates $(16 \times 16 \mathrm{~cm})$, which remained before establishing bacterial separates. Holes were occupied with $100 \mathrm{ml}$ of the established product concentration $(100 \mathrm{mmol}$ disbanded in $1 \mathrm{ml}$ DMSO) (Sunita and Mahendra, 2008; Andrews, 2001; Abdou et al., 2014). Subsequently, the dish protected for $24 \mathrm{~h}$ at $37^{\circ} \mathrm{C}$. Subsequently maturation, the antimicrobiogical action of every regular product was estimated through determining the inhibition region diameters in contrast to examine bacteria and associated with typical region ranges of their standard sulfa medication. The experimentation was accomplished in triplicate and the regular region of inhibition was premeditated. Primarily, totally manufactured products and standard drugs Amphotericin $B$, Ampicillin and Gentamicin were estimated in vitro for their antimicrobiogical action, through the inhibition region procedure, exhausting three $\operatorname{Gram}(+)$ bacteria: $S$. pneumonia (RCMB 010010), Enterococcus faecalis (RCMB 010068) and S. aureus (RCMB 010028) through the accumulation of three Gram(-) bacteria: E. coli (RCMB 010052) and Salmonella typhimurium (RCMB 010072) and Pseudomonas aeruginosa (RCMB 010043), also were estimated in vitro for their antifungial action through the inhibition region procedure in contrast to Candida albicans (RCMB 05079) and Aspergillus fumigatus (RCMB 02568).

Inhibition region diameter acquired for resultant products recommends that totally manufactured products retain noteworthy antimicrobiolgical action in contrast to greatest examined organisms used in these evaluates (Table 3), Compounds $4 \mathbf{e}, \mathbf{4 f}, \mathbf{4 g}, \mathbf{4 k}, \mathbf{4 l}, \mathbf{4 o}$ and $\mathbf{4 p}$ demonstrated higher antibacterial and antifungal. In addition, Compounds $4 \mathrm{~d}, 4 \mathrm{~h}, 4 \mathrm{~m}$ and $4 \mathrm{n}$ showed moderate activity. For the optimization purpose, the most active agents $4 \mathbf{o}, \mathbf{4 p}, \mathbf{4 e}, \mathbf{4 f}, \mathbf{4 g}$ and $\mathbf{4 h}$ to all strains was designated for further modification, anticipating increasing the antimicrobial along with the antimycobacterial activities due to withdrawing group or a heterocyclic group. Contrariwise, compounds $4 \mathbf{k}, \mathbf{4 l}, \mathbf{4 m}$ and $4 \mathrm{n}$ are exactly consuming the same activity. It is value mentioning that interaction of electron withdrawing group or heterocyclic groups in $4 \mathrm{e}, 4 \mathrm{f}, 4 \mathrm{~g}$,
$4 \mathrm{o}$ and $4 \mathrm{p}$ manufactured a high antimicrobial activity than electron donating group. Compounds $4 \mathrm{a}, 4 \mathrm{~b}, 4 \mathrm{c}, 4 \mathrm{~d}$, $4 \mathrm{i}$ and $4 \mathrm{j}$ exhibited moderate activities alongside all strains; these results designate that additional donating group's substituents reduces the antimicrobial activity. Nevertheless, the highest activity obtained from 2amino-4-(4-chlorophenyl)-3-(methoxycarbonyl)-4H-

pyrano[2,3-c]pyridine-8-carboxylic acid $(4 \mathrm{e})$ and 2amino-3-cyano-4-(pyridin-4-yl)-4H-pyrano[2,3-

c]pyridine-8-carboxylic acid (4p) and 2-amino-3-cyano4-(4-nitrophenyl)-4H-pyrano[2,3-c]pyridine-8-

carboxylic acid (4l) groups highest against $S$. pneumonia and higher also against Aspergillus fumigatus .

\section{Free Radical Scavenging Action}

The radical scavenging action of the manufactured products was confirmed by DPPH technique. Free radical (DPPH) is admitted one electron or hydrogen radical to come to be established diamagnetic fragment. DPPH in methanol appearances a characteristic band at $517 \mathrm{~nm}$ (dependent of PH beginning 5.0 to 6.5) and the solution performs to be bottomless violet color. For instance, DPPH radical is going through the donation hydrogen from the antioxidant, the point of staining designates the searching potential of the antioxidant products. Temporarily, different solution concentration $\left(100,200,300,400,500 \mu \mathrm{g} \mathrm{ml}^{-1}\right)$ of the examine products and ascorbic acid (standard) were organized in methanol and supplementary $(1.5 \mathrm{ml})$ to the methanolic solution of DPPH $(1.5 \mathrm{ml}, 200 \mu \mathrm{M})$ (Abu-Hashem et al., 2011; Mohamed et al., 2012 and Shu et.al 2007). The mix was stunned forcefully and permitted to attitude for 30 min in the dark. Subsequently, this, the absorbance was tested at $517 \mathrm{~nm}$. Methanol $(1.5 \mathrm{ml})$ was diversified with DPPH solution $(1.5 \mathrm{ml}, 200 \mu \mathrm{M})$. The scavenging action percentage was designed exhausting formulation:

$$
\% \text { Inhibition }=(A c-A t / A c) \times 100
$$

Where, the Ac = observance control $(1.5 \mathrm{ml}$ of each of methanol and the $200 \mu \mathrm{M}$ DPPH solution), At $=$ absorption test compound/ascorbic acid.

The inhibition percentage (\%) curvatures for ascorbic acid and compounds were strategized in contrast to the concentration, from which $\mathrm{IC}_{50}$ values of the inhibition percentage of DPPH via ascorbic acid and samples were considered exhausting regression equation.

The synthesized samples were selected for in-vitro antioxidant action by DPPH technique. The data achieved are represented in Table 4 as $\mathrm{IC}_{50}(\mu \mathrm{M})$ values and supplementary to those of ascorbic acid as typical. 
Improved observance of the samples with concentration exposes that products retain the radical scavenging action. Analysis of the results in Table 4. The manufactured products were selected for in vitro antioxidant action through DPPH technique. The data acquired are represented in Table 4 as $\mathrm{IC}_{50}(\mu \mathrm{M})$ values and paralleled with those of ascorbic acid as typical. Absorbance increasing of the products with concentration exposes that products retain radical scavenging action. Analysis of the results in Table 2 which indicated the insertion of electron donating $\mathrm{CH}_{3}$ and $\mathrm{OCH}_{3}$ groups, as $4 \mathrm{c}, 4 \mathrm{~d}, 4 \mathrm{i}$ and $4 \mathrm{j}$ reduced the radical scavenging activity and the electron withdrawing $\mathrm{Cl}, \mathrm{Br}$ and $\mathrm{NO}_{2}$, as in $4 \mathrm{e}, 4 \mathrm{f}, 4 \mathrm{~g}, 4 \mathrm{~h}, 4 \mathrm{k}$ and $4 \mathrm{l}$ increase the radical scavenging, moreover, pyrano[2,3-c]pyridine group of $4 m, 4 n, 4 o, 4 p$ encourages an growth in the antioxidant property. Among the products experienced $4 \mathrm{o}$ and $4 \mathrm{p}$ demonstrated effective free radical scavenging action with $\mathrm{IC}_{50}$ standards of 252.52 and $223.2 \mu \mathrm{M}$, respectively (Morimoto et al., 1995).

The indication of the data obtained in Table 3 and 4 exposed that, generally pyrano[2,3-c]pyridine accompanying to heterocyclic were further active than those enclosing aromatic rings. Further studies are desirable to be supported out to invention association between $\mathrm{IC}_{50}$ of the evaluated pyrano[2,3-c]pyridine and their molecular descripts, for instance electronic, lipophilic and steric parameters.

Table 3: Antimicrobial activity $(\mathrm{mg} / \mathrm{ml})$ of compounds 4a-p

\begin{tabular}{|c|c|c|c|c|c|c|c|c|}
\hline Compound & $\begin{array}{l}\text { S.pneumonia } \\
\text { (RCMB } \\
010010 \text { ) }\end{array}$ & $\begin{array}{l}\text { Enterococcus } \\
\text { faecalis (RCMB } \\
010068)\end{array}$ & $\begin{array}{l}\text { S. aureus } \\
\text { (RCMB } \\
010028)\end{array}$ & $\begin{array}{l}\text { E.coli } \\
\text { (RCMB } \\
010052)\end{array}$ & $\begin{array}{l}\text { Salmonella } \\
\text { typhimurium } \\
\text { (RCMB 010072) }\end{array}$ & $\begin{array}{l}\text { Pseudomonas } \\
\text { aeruginosa } \\
\text { (RCMB 010043) }\end{array}$ & $\begin{array}{l}\text { Candida } \\
\text { albicans } \\
\text { (RCMB 05079) }\end{array}$ & $\begin{array}{l}\text { Aspergillus } \\
\text { fumigates } \\
\text { (RCMB 02568) }\end{array}$ \\
\hline $4 a$ & $19.7 \pm 0.25$ & $\mathrm{NA}^{*}$ & $10.2 \pm 0.51$ & $8.3 \pm 0.58$ & $11.3 \pm 0.36$ & NA & $11.3 \pm 0.25$ & $12.5 \pm 0.19$ \\
\hline $4 b$ & $17.7 \pm 0.19$ & $12.5 \pm 0.44$ & $9.2 \pm 0.32$ & $11.6 \pm 0.42$ & NA & $10.3 \pm 0.37$ & $12.7 \pm 0.44$ & $14.6 \pm 0.37$ \\
\hline $4 c$ & $12.0 \pm 0.25$ & $10.6 \pm 0.37$ & $10.3 \pm 0.55$ & $11.8 \pm 0.57$ & $15.9 \pm 0.44$ & $11.9 \pm 0.25$ & $11.4 \pm 0.34$ & $12.6 \pm 0.37$ \\
\hline 4d & $18.9 \pm 0.25$ & $16.8 \pm 0.19$ & $10.5 \pm 0.54$ & $12.3 \pm 0.21$ & $15.9 \pm 0.44$ & $14.9 \pm 0.58$ & $13.2 \pm 0.37$ & $18.7 \pm 0.37$ \\
\hline $4 e$ & $23.5 \pm 0.4$ & $19.5 \pm 0.44$ & $14.3 \pm 0.25$ & $16.8 \pm 0.42$ & $16.9 \pm 0.36$ & $16.8 \pm 0.58$ & $15.4 \pm 0.19$ & $20.6 \pm 0.19$ \\
\hline $4 f$ & $21.5 \pm 0.25$ & $18.7 \pm 0.58$ & $16.3 \pm 0.15$ & $17.3 \pm 0.49$ & $15.24 \pm 0.44$ & $14.8 \pm 0.37$ & $16.3 \pm 0.25$ & $18.8 \pm 0.44$ \\
\hline $4 g$ & $20.3 \pm 0.27$ & $17.3 \pm 0.58$ & $18.3 \pm 0.25$ & $19.3 \pm 0.25$ & $16.2 \pm 0.58$ & $15.2 \pm 0.25$ & $18.4 \pm 0.44$ & $19.3 \pm 0.44$ \\
\hline $4 h$ & $17.5 \pm .37$ & $19.3 \pm 0.44$ & $16.3 \pm 0.25$ & $17.4 \pm 0.31$ & $14.3 \pm 0.25$ & $16.4 \pm 0.25$ & $19.4 \pm .44$ & $15.8 \pm 0.44$ \\
\hline $4 i$ & $14.3 \pm 0.58$ & $15.3 \pm 0.63$ & $7.3 \pm 0.42$ & $15.3 \pm 0.28$ & $13.7 \pm 0.42$ & $14.8 \pm 0.19$ & $17.8 \pm 0.63$ & $14.6 \pm 0.37$ \\
\hline $4 j$ & $14.9 \pm 0.25$ & $11.7 \pm 0.37$ & $10.3 \pm 0.35$ & $14.3 \pm 0.37$ & $14.2 \pm 0.37$ & $11.4 \pm 0.25$ & $11.7 \pm 0.19$ & $12.9 \pm 0.19$ \\
\hline $4 k$ & $23.4 \pm 0.37$ & $19.1 \pm 0.25$ & $11.3 \pm 0.37$ & $20.9 \pm 0.58$ & $15.6 \pm 0.58$ & $17.8 \pm 0.25$ & $20.9 \pm 0.25$ & $19.8 \pm 0.19$ \\
\hline 41 & $22.3 \pm 0.44$ & $17.2 \pm 0.19$ & $12.3 \pm 0.4$ & $15.8 \pm 0.19$ & $12.6 \pm 0.42$ & $13.3 \pm 0.25$ & $14.7 \pm 0.58$ & $16.2 \pm 0.44$ \\
\hline $4 m$ & $19.8 \pm 0.25$ & $\mathrm{NA}^{*}$ & $12.3 \pm 0.25$ & $13.6 \pm 0.52$ & $13.7 \pm 0.44$ & NA & $11.3 \pm 0.25$ & $14.5 \pm 0.41$ \\
\hline $4 n$ & $19.3 \pm 0.19$ & $17.8 \pm 0.44$ & $12.4 \pm 0.28$ & $16.7 \pm 0.58$ & $14.8 \pm 0.37$ & $16.8 \pm 0.58$ & $18.7 \pm 0.44$ & $20.6 \pm 0.19$ \\
\hline 40 & $20.5 \pm 0.25$ & $0.37 \pm 18.2$ & $17.2 \pm 0.19$ & $17.8 \pm 0.23$ & $13.8 \pm 0.63$ & $17.3 \pm 0.37$ & $17.7 \pm 0.44$ & $15.3 \pm 0.25$ \\
\hline $4 p$ & $22.3 \pm 0.25$ & $0.19 \pm 17.2$ & $12.6 \pm 0.25$ & $18.3 \pm 0.25$ & $16.2 \pm 0.44$ & $14.9 \pm 0.58$ & $19.2 \pm 0.37$ & $18.7 \pm 0.37$ \\
\hline Amphotericin B & $25.4 \pm 0.1$ & $28.7 \pm 0.2$ & $19.7 \pm 0.2$ & $23.7 \pm 0.1$ & - & - & - & - \\
\hline Ampicilline & - & - & - & - & - & - & $23.8 \pm 0.2$ & $32.4 \pm 0.3$ \\
\hline Gentamicin & - & - & - & - & $17.3 \pm 0.1$ & $19.9 \pm 0.3$ & - & - \\
\hline
\end{tabular}

NA*: No Action, \pm Standard Deviation

Table 4: Free radical scavenging action of the manufactured products utilizing DPPH technique

\begin{tabular}{lll}
\hline Compound & Inhibition & \\
\hline $\mathbf{4 a}$ & $58.23 \%$ & $\mathrm{IC}_{50} \pm \mathrm{SE}^{\mathrm{a}}(\mu \mathrm{M})$ \\
$\mathbf{4 b}$ & $61.25 \%$ & $783.22 \pm 4.8$ \\
$\mathbf{4 c}$ & $45.36 \%$ & $652.42 \pm 7.85$ \\
$\mathbf{4 d}$ & $52.3 \%$ & $587.23 \pm 6.7$ \\
$\mathbf{4 e}$ & $77.68 \%$ & $409.23 \pm 5.86$ \\
$\mathbf{4 f}$ & 78.66 & $509.26 \pm 3.32$ \\
$\mathbf{4 g}$ & 82.52 & $423.52 \pm 6.37$ \\
$\mathbf{4 h}$ & $79.68 \%$ & $512.17 \pm 5.11$ \\
$\mathbf{4 i}$ & $43.22 \%$ & $815.71 \pm 3.01$ \\
$\mathbf{4 j}$ & 58.235 & $958.06 \pm 8.09$ \\
$\mathbf{4 k}$ & $68.91 \%$ & $574.2 \pm 3.88$ \\
$\mathbf{4 1}$ & $66.23 \%$ & $479.18 \pm 6.23$ \\
$\mathbf{4 m}$ & $67.9 \%$ & $385.32 \pm 3.32$ \\
$\mathbf{4 n}$ & $65.23 \%$ & $362.55 \pm 4.5$ \\
$\mathbf{4 0}$ & $83.3 \%$ & $252.53 \pm 5.82$ \\
$\mathbf{4 p}$ & $88.6 \%$ & $223.2 \pm 3.88$ \\
Ascorbic acid & $90.20 \%$ & $100.2 \pm 9.6^{\mathrm{b}}$ \\
\hline
\end{tabular}

${ }^{\mathrm{b}} \mathrm{IC}_{50}$ values represent as mean $\pm \mathrm{SD}$ of three determinations 


\section{Conclusion}

We require conceived a green and effective simple technique for the production pyrano[2,3-c]pyridine derivatives beneath DMAP catalyst exhausting microwave and conventional irradiation. The investigational ease, short reaction periods, extraordinary incomes, easy workup procedures, prevention of organic solvents and consumption of an expensive and freely obtainable and wastefully smart to synthesis these compounds. The improvement of DMAP in contrast to recognized catalyst is (i) cheap, (ii) eco-friendly and (iii) no essential chromatographic separation. The manufactured compounds exhibited moderate to good in vitro antimicrobial and antioxidant activities when associated with standard drugs. Compounds $4 \mathrm{e}, 4 \mathrm{o}$ and $4 \mathrm{p}$ demonstrated high potency against antimicrobial or antioxidant due to incorporated two heterocyclic moieties.

\section{Acknowledgement}

The author acknowledges the support of this study of the Green Chemistry Department and National Research Centre Egypt. Many thanks to the staff of the Micro analytical Centre of Al Azhar University at which all analyses were made.

\section{Ethics:}

Author declared no conflict of interests.

\section{References}

Abdou, W.M., N.A. Ganoub and R.F. Barghash, 2014. Synthesis and bioactivity of benzothiazaphosphepines and relevant phosphonates as antioxidant/antidiabetic agents. Synthetic Communic.

Abu-Hashem, A.A., M.M. Youssef and H.A.R. Hussein, 2011. Synthesis, antioxidant, antituomer activities of some new thiazolopyrimidines, pyrrolothiazolopyrimidines and triazolo pyrrolo thiazolopyrimidines derivatives. J. Chem. Society, 58: 41-48.

Akbar, M., F. Naser, M. Hassan and K. Nahid, 2015. Piperazine catalyzed convenient synthesis of $4 \mathrm{H}-$ pyran derivatives from $\alpha, \alpha^{\prime}$-bis(substitutedbenzylidene) cycloalkanones and malononitrile under reflux conditions. J. Saudi Chem. Society.

Andrews, J.M., 2001. Determination of minimum inhibitory concentrations. J. Antimicrob Chemother, 48: 5-16.

Armand, G., K. Charline P. Nicolas, L. Gilles and G. Michel et al., 2014. A new DMAP-catalyzed and microwave-assisted approach for introducing heteroarylamino substituents at position 4 of the quinazoline ring. Tetrahedron, Elsevier, 78: 82578266 .
Ashraf, H.F.A.E.W, 2012. Synthesis, reactions and evaluation of the antimicrobial activity of some 4(p-halophenyl)-4h-naphthopyran, pyranopyrimidine and pyranotriazolopyrimidine derivatives. Pharmaceuticals (Basel), 5: 745-757.

Bonsignore, L., G. Loy, D. Secci and A. Calignano, 1993. Synthesis and pharmacological activaty of 2Oxo-(2H)-1- benzopyran-3-carboxamide Derivatives. Eur. J. Med. Chem., 28: 517-517.

Brahmachari, G., S. Laskar and B. Banerjee, 2014. Ecofriendly, one-pot multicomponent synthesis of pyran annulatedheterocyclic scaffolds at room temperature using ammonium or sodium formate as non-toxic catalyst. J. Heterocyclic Chem., 51: 303-303.

Dean, F.M.,1963. Naturally Occurring Oxygen Ring Compounds. 1st Edn., Butter-Worths, London.

Dinesh, K., S. Pooja, S. Harmanpreet, N. Kunal and K. Girish et al., 2017. The value of pyrans as anticancer scaffolds in medicinal chemistry. RSC Adv., 7: 36977-36999.

Dömling, A. and I. Ugi, 2000. Multicomponent reactions with isocyanides. Angew. Chem., Int. Ed., 39: 3168-3210.

Dömling, A., 2006. Recent developments in isocyanide based multicomponent reactions in applied chemistry. Chem. Rev., 106: 17-89. DOI: 10.1021/cr0505728

Elham, S.D., F.A. Fattah, F.A. Attaby and O.N. AlShayea, 2014. Synthesis and antimicrobial evaluation of some novel thiazole, pyridone, pyrazole, chromene, hydrazone derivatives bearing a biologically active sulfonamide moiety. Int. J. Mol. Sci., 215: 1237-1254. DOI: 10.3390/ijms15011237

Fan, X., D. Feng, Y. Qu, X. Zhang and J. Wang et al., 2010. Practical and efficient synthesis of pyrano[3,2-c] pyridone, pyrano[4,3-b]pyran and their hybrids with nucleoside as potential antiviral and antileishmanial agents, Bioorg. Med. Chem. Lett., 20: 809-813.

Feuer, G., 1974. Progress in Medicinal Chemistry. In: North-Holland Publishing Company, Ellis, G.P. and G.P. West, (Eds.), Elsevier, New York, ISBN-10: 0080862616 , pp: 356.

Jitender, M.K. and C. Ankita, 2012. Efficient and green synthesis of 4H-pyrans and 4H-pyrano[2,3-c] pyrazoles catalyzed by task-specific ionic liquid [bmim] $\mathrm{OH}$ under solvent-free conditions. Green Chem. Lett. Rev., 5: 633-638.

Karnakar, K., K. Ramesh, K.H.V. Reddy, K.B.S.P. Anil and N.J. Babu et al., 2015. A novel one pot fourcomponent reaction for the efficient synthesis of spiro[indoline-3,4'-pyrano[2,3-c]pyrazole]-3'carboxylate and trifluoro methylated spiro[indole3,4'-pyrano[2,3-c]pyrazole] derivatives using recyclable PEG-400. New J. Chem., 39: 8978-8983.

Khan, A.T. and M.M. Khan, 2011. One-pot threecomponent reaction for the synthesis of pyran annulated heterocyclic compounds using DMAP as a catalyst. Tetrahedron Lett., 52: 3455-3455. 
Khan, A.T., M. Lal and M.M. Khan, 2010 a. Synthesis of highly functionalized piperidines by one-pot multicomponent reaction using Tetrabutylammonium Tribromide (TBATB). Tetrahedron Lett., 51: 4419-4419.

Khan, A.T., M.M. Khan and K.K.R. Bannuru, 2010. b. Iodine catalyzed one-pot five-component reactions for direct synthesis of densely functionalized piperidines. Tetrahedron, 66: 7762-7762.

Khan, A.T., T. Parvin and L.H. Choudhury, 2008. Effects of substituents in the $\beta$-position of 1,3 dicarbonyl compounds in bromodimethylsulfonium bromide-catalyzed multicomponent reactions: A facile access to fun. J. Org. Chem., 73: 8398-8398.

Mady, M.F., T.S. Saleh, A.A. El-Kateb, N.M. Abd ElRahman and S.I. Abd El-Moez, 2015. Microwaveassisted synthesis of novel pyrazole and pyrazolo[3,4-d]pyridazine derivatives incorporating diaryl sulfone moiety as potential antimicrobial agents. Res. Chem. Intermediates, 42: 753-769.

Mohamed, M., 1. Youssef and M.A. Amin, 2012. Microwave assisted synthesis of some new thiazolopyrimidine, thiazolodipyrimidine and thiazolopyrimidothiazolopyrimidine derivatives with potential antioxidant and antimicrobial activity. Molecules, 17: 9652-9667.

Morimoto., Y., K. Tanaka, Y. Iwakiri, S. Tokuhiro and S. Fukushima et al., 1995. Protective effects of some neutral amino acids against hypotonic hemolysis. Pharm. Bull., 18: 1417-1422.

Nandakumar, A., P. Thirumurugan, P.T. Perumal, P. Vembu and M.N. Ponnuswamy et al., 2010. One-pot multicomponent synthesis and antimicrobial evaluation of 2'-(indol-3-yl)-2-oxospiro(indoline3,4'-pyran) derivatives. Bioorg. Med. Chem. Lett., 20: 4252-4258.

Saeed, B., B. Morteza, S.A. Masoumeh, H. Shohreh and S. Peyman, 2007. Diammonium hydrogen phosphate: An efficient and versatile catalyst for the one-pot synthesis of tetrahydrobenzo [b] pyran derivatives in aqueous media. Synthetic Commun., 8: 1724-1728.
Saeed, K.I., M. Farzaneh, R. Alimorad and M.K. Abbasabadi, 2015. A green synthesis of substituted coumarins using nano graphene oxide asrecyclable catalyst. J. Chin. Chem. Soc., 62: 000-000.

Salem, M.E., A.A. Ahmed, M.R. Shaaban, M.F. Shibl and A.M. Farag, 2015. Regioselective synthesis and $\mathrm{ab}$ initio calculations of fused heterocycles thermally and under microwave irradiation. Spectrochimica Acta Part A: Molecular Biomolecular Spectroscopy, 148: 175-183. DOI: 10.1016/j.saa.2015.03.102

Shu, Y., S.A. Sheardown, C. Brown, R.P. Owen and S. Zhang et al., 2007. Effect of genetic variation in the organic cation transporter 1 (OCT1) on metformin action. J. Clin. Invest., 117: 1422-1431.

Sunita, B. and R. Mahendra, 2008. Antifungal Activity of Essential Oils from Indian Medicinal Plants Against Human Pathogenic Aspergillus fumigatus and A. niger. World J. Med. Sci., 93: 81-88.

Thomas, J.J.M., 2017. Multicomponent reactions in the synthesis of heterocycles. Chem. Heterocyclic Compounds, 53: 381-381.

Vyas, D.H., S.D. Tala, J.D. Akbai, M.F. Dhaduk and K.A. Joshi et al., 2009. Synthesis and antimicrobial activity of some new cyanopyridine and cyanopyrans towards Mycobacterium tuberculosis and other microorganisms. Ind. J. Chem., 48B: 833-839.

Wen, C., S.D. Shieh and R. Oljan, 2001. 1,8Diazabicyclo[5.4.0] undec-7-ene (DBU) and microwave-accelerated green chemistry in methylation of phenols, indoles and benzimidazoles with dimethyl carbonate. Org. Lett., 3: 4279-4281.

Yusuke, J., K. Masato and N. Jiro, 2016. Phase transition of tetra-n-butylammonium bromide hydrates enclosing krypton. J. Chem. Eng. Data, 61: 679-685.

Zhu, J. and H. Bienayme, 2005. Multicomponent Reactions. 1st Edn., Wiley-VCH: Weinheim, Germany.

Zhu, J., 2003. Recent developments in the isonitrilebased multicomponent synthesis of heterocycles. J. Eur. J. Org. Chem. 